\title{
Estudio comparativo de las propiedades fisicoquímicas y microbiológicas de tres tipos de lactosuero (ovino, bovino y caprino) para uso alimentario.
}

Comparative study of physicochemical and microbiological properties of three types of whey (bovine, ovine and caprine) for food use.

Nora Tahirí Mejía Cabezas. ${ }^{1}$, Diana Katherine Campoverde Santos. ${ }^{2}$ \& Luis Antonio Velasco Matveev. ${ }^{3}$

\begin{abstract}
.
DOI: https://doi.org/10.33262/concienciadigital.v4i3.1.1831

Introduction. Whey is a by-product of cheese production, containing mainly lactose, proteins, minerals, vitamins and fat. Its composition varies considerably depending on the type of milk, type of cheese produced and the technological process used, but in general it represents between $85 \%$ and $95 \%$ of the volume of milk and retains $55 \%$ of its nutrients. The amount of whey obtained for each kilogram of cheese is approximately 9 liters, which is why its proper use is of great importance. Objective. The objective of the present study was to evaluate three types of whey: bovine, ovine and caprine. Methodology. The analyzes were carried out in a bromatological laboratory of a private company, where physical variables such as $\mathrm{pH}$ and density were analyzed; chemical variables such as dry extract, ash, protein, fat, calcium and, lactose; also microbiological variables such as the

1 Escuela Superior Politécnica de Chimborazo, Facultad de Ciencias Pecuarias, Ingeniería en Agroindustrias, Chimborazo, Riobamba, Ecuador, nora.mejia@espoch.edu.ec, https://orcid.org/00000002-0308-5412.

2 Escuela Superior Politécnica de Chimborazo, Facultad de Ciencias Pecuarias, Medicina Veterinaria, Chimborazo, Riobamba, diana.campoverde@espoch.edu.ec, Ecuador, https://orcid.org/0000-00018538-6747.

3 Escuela Superior Politécnica de Chimborazo, Facultad de Ciencias Pecuarias, Ingeniería Zootécnica Riobamba, Ecuador, lvelasco@espoch.edu.ec, https://orcid.org/0000-0002-4497-576.
\end{abstract}


coliform population. Results. Significant differences were obtained at $\mathrm{p}<0.05$ and at $\mathrm{p}$ $<0.01$ in the samples. Sheep whey was the one that registered the best results for total solids with $7.48 \%$, in proteins it contributed $1.05 \%$ and, fats with $0.73 \%$. Calcium, $\mathrm{pH}$, density and, lactose did not show significant differences between the wheys analyzed. The goat whey presents coliform levels of $1.89 \log 10 \mathrm{UFC} \cdot \mathrm{ml}-1$, being higher than the others. Conclusion. It was concluded that sheep whey is the most suitable for the production of protein concentrates, food and also for the pharmaceutical industry.

Keywords: Whey, goat, sheep, bovine.

\section{Resumen.}

Introducción. El lactosuero es un subproducto que resulta de la elaboración del queso, contiene principalmente lactosa, proteínas, minerales, vitaminas y grasa, su composición varía considerablemente dependiendo del tipo de leche, tipo de queso elaborado y el proceso de tecnología empleado, pero en general representa entre el $85 \%$ al $95 \%$ del volumen de leche y retiene el $55 \%$ de sus nutrientes. La cantidad de suero obtenida por cada kilogramo de queso es de 9 litros aproximadamente, motivo por el cual darle un uso adecuado es de gran relevancia. Objetivo. El objetivo del presente estudio fue evaluar tres tipos de lactosuero: bovino, ovino y caprino. Metodología. El análisis estadístico utilizado fue el diseño completamente al azar. Las pruebas se realizaron en un laboratorio bromatológico de una empresa privada, en donde se analizaron variables físicas como el pH y la densidad; variables químicas como el extracto seco, cenizas, proteínas, grasas, calcio, lactosa y una variable microbiológica denominada como población de coliformes. Resultados. Se obtuvieron diferencias significativas al $\mathrm{p}<0.05$ y al $\mathrm{p}<0.01$ en las muestras. El lactosuero ovino fue el que mejores resultados registró para sólidos totales con un $7.48 \%$, en proteínas aporta un $1.05 \%$ y grasas con un $0.73 \%$. El calcio, pH, densidad y lactosa no presentaron diferencias significativas entre los sueros. El suero caprino presenta niveles de coliformes de $1.89 \log 10 \mathrm{UFC} \cdot \mathrm{ml}-1$, siendo superior a los otros. Conclusión. Se concluyó que el lactosuero ovino es el más adecuado para la elaboración de concentrados proteicos, alimentos y también para la industria farmacéutica.

Palabras claves: Lactosuero, caprino, ovino, bovino.

\section{Introducción.}

El suero de leche o lactosuero es un subproducto que resulta de la elaboración del queso, el cual es obtenido después de la precipitación de la caseína, contiene principalmente lactosa, proteínas como sustancias de importante valor nutritivo, minerales, vitaminas y grasa. La composición y tipo de lactosuero varía considerablemente dependiendo del tipo de leche, tipo de queso elaborado y el proceso de tecnología empleado.(Adolfo \& Huertas, 2008) 
Existen dos tipos de suero, el lactosuero ácido con un $\mathrm{pH}$ de 4.5 y el lactosuero dulce que tiene un pH de entre 6.0 y 6.5 (Gómez Soto \& Sánchez Toro, 2019). Los lactosueros dulces surgen de la fabricación de quesos frescos de pasta blanda, obtenidos a partir de leche de vaca y/o de cabra; en ellos, la lactosa se ha transformado en ácido láctico, son ricos en calcio y fósforo, en cambio un lactosuero dulce, proviene de la fabricación de quesos de pasta cocida y prensada a partir de leche vaca y oveja; es pobre en ácido láctico (Callejas Hernández et al., 2012). La cantidad de suero obtenida por cada kilogramo de queso es de 9 litros aproximadamente, motivo por el cual, darle un uso adecuado es de gran relevancia. (Onwulata \& Huth, 2008)

El lactosuero representa aproximadamente, del $85 \%$ al 95\% del volumen de leche, y retiene el 55\% de sus nutrientes. Estos nutrientes pueden recuperarse para ser utilizados en diferentes campos como los alimentarios y farmacéuticos. (Siso, 1996). La lactosa es el principal componente nutritivo $(4.5 \%$ p-v), proteína $(0.8 \%$ p/v), y lípidos $(0.5 \%)$. (Adolfo \& Huertas, 2008)

El lactosuero casi siempre ha sido considerado como un desecho con poco valor comercial. Su destino sigue siendo uno de los problemas más serios que enfrenta la industria láctea a nivel mundial. (Araujo Guerra et al., 2013) En el mercado existen productos que incluyen al lactosuero como ingrediente, principalmente como medio para aumentar los sólidos lácteos a un bajo costo y, en menor grado, para aprovechar algunas de sus propiedades funcionales de las proteínas del suero. (Alvarado Carrasco \& Guerra, 2010)

Para el año 2017, la producción de queso a nivel mundial se estimó en 20015000 toneladas. Se calcula que esta cantidad de queso produjo 180135000 toneladas de lactosuero aproximadamente (Huertas, 2009)

Para el año 2016 se produjeron en el mundo 21800 toneladas de leche de cabra y 13800 toneladas de leche ovina. De esto el $90 \%$ se utiliza para preparar quesos (Ghibaudi et al., 2018; INEC, 2002)

Ecuador destina 1.5 millones de litros de leche para la producción formal de 300000 kilos de quesos por día. Eso genera 1.2, millones de litros de suero, aunque solo el 10\% de este total estaría usándolo la industria para fabricar en su mayoría bebidas lácteas. (el Universo, 2019)

Este suero es suficiente para alimentar a 120000 personas por día. Sin embargo, debido a los acuerdos ministeriales gran parte de este producto no se comercializa y termina desperdiciándose como comida para cerdos de traspatio y vertiéndose sobre el sistema de alcantarillado público o quebradas. El desperdicio del suero genera un grave problema ambiental que va en contra de las iniciativas mundiales relacionadas a los objetivos de Desarrollo Sostenible, Economía Circular, entre otros.

El martes, 17 de septiembre de 2019, la Asamblea Nacional introdujo entre las reformas al Código Orgánico Integral Penal (COIP) la sanción a la oferta o venta del suero de leche 
con fines comerciales en la cadena láctea, excepto si se encuentra concentrado. ( https://www.primicias.ec/noticias/economia/suero-leche-industria-agronomialactosuero/)

En general, resulta interesante mirar las propiedades existentes en la leche de una amplia gama de mamíferos para comparar las variaciones en composición entre las diferentes especies. La mayoría de los sueros se generan a partir del procesamiento de las leches de vaca, oveja y cabra, porque son las de mayor importancia a nivel mundial, siendo la leche de vaca la más producida y utilizada. (Zadow, 1992)

En el presente estudio se analizarán las propiedades fisicoquímicas y microbiológicas del lactosuero ovino, bovino y caprino. Materia seca, cenizas, proteína, lactosa, grasa, calcio, acidez, fosfatasa, $\mathrm{pH}$ y densidad, son aquellas catalogadas como propiedades fisicoquímicas, mientras que la cantidad coliformes presentes será el parámetro establecido para hablar sobre sus propiedades microbiológicas.

El lactosuero contiene muchas sustancias biológicamente activas, que pueden actuar como agentes antibacterianos y antivirales modificadores, además contiene varios componentes que pueden proteger contra toxinas, bacterias y virus. Estos componentes incluyen inmunoglobulinas, calostro, lactoferrinas (LF) y sus derivados peptídicos, como lactoferrina, lactoperoxidasa, glicomacropéptidos (GMP) y esfingolípidos (Floris et al., 2003; Shukla et al., 2017). Es más, los péptidos antimicrobianos se pueden generar a partir de proteína de suero por proteólisis durante el tránsito gastrointestinal.

El papel antimicrobiano de las LF es de particular interés para la función intestinal y la prevención de enfermedades gastroentéricas mediante el control de la microflora intestinal. Mientras que LF exhibe actividad bactericida contra patógenos como coliformes, también proporciona soporte probiótico para microorganismos beneficiosos como Bifidobacteria y Lactobacilli ssp. (Yamauchi et al., 2006), e incluso varios estudios han reportado que la administración diaria de LF suprime positivamente la colonización intestinal de Helicobacter pylori, el cual se conoce como agente causal en la mayoría de las úlceras duodenales y se cree que es responsable del 50\% -60\% de todos los carcinomas gástricos (Collins et al., 2006).

En la actualidad, el principal uso para el lactosuero es en la industria alimentaria, para elaborar concentrados o aislados proteicos, suplementos y un sin número de productos con un alto valor nutricional, debido a la gran producción de leche de vaca, la cual es conocida como un alimento de primera necesidad, la mayor cantidad de investigaciones relativas al tema se enfocan en el estudio del lactosuero bovino, a diferencia de lo que ocurre con el suero bovino, de los cuales existe poca información disponible.

\section{Metodología.}

El estudio se realizó en un laboratorio bromatológico de una empresa privada, para ello se utilizaron 3 muestras de suero de distintas especies (ovino, caprino y bovino) con la finalidad de determinar las propiedades fisicoquímicas (extracto seco, cenizas, proteína, 
lípidos o grasas, calcio, lactosa, $\mathrm{pH}$, densidad) y microbiológicas del lactosuero. A continuación, se detalla el proceso de determinación de cada variable:

\section{Extracto Seco}

El extracto seco es todo lo que queda una vez eliminada la humedad del suero. Se utilizó la desecación del suero en estufa a una temperatura de $105^{\circ} \mathrm{C}$. Previo al análisis la muestra fue homogenizada para seguir el proceso correspondiente. En primer lugar, se determinó la humedad, pesando las muestras líquidas y las sólidas una vez realizado el proceso de desecado.

El contenido seco se calculó restando el contenido líquido del peso total de la muestra y se expresó el extracto seco en función de porcentaje mediante la fórmula

$$
\text { Extracto } \operatorname{seco}(\%)=\frac{\text { pesofinal }}{\text { peso } \text { inicial }} \times 100
$$

\section{Cenizas}

Se incineraron muestras de cada tipo de lactosuero exactamente pesadas en un crisol de porcelana utilizando una mufla a una temperatura de $500^{\circ}$ durante 7 horas. Cuando la muestra se encuentra libre de partículas carbonosas y las cenizas presentan un color blanco o gris uniforme, el proceso se da por terminado y se pesa utilizando una balanza analítica para obtener resultados precisos. Al igual que el contenido de extracto seco, el contenido de cenizas se expresa en porcentaje.

\section{Proteína}

La cantidad de proteínas existente en cada una de las muestras de suero se determinó mediante precipitación por sales. Una vez obtenido el precipitado, se utilizó el método gravimétrico para obtener un valor exacto y el resultado se expresó en porcentaje.

\section{Lípidos o grasas}

Los lípidos en las muestras de lactosuero fueron obtenidos mediante butirometría. Para separar las grasas se colocó ácido sulfúrico y posteriormente se llevó a cabo un proceso de centrifugación, que evita la carbonización y finalmente se desenmulsionaron las grasas se añade alcohol amílico.

\section{Calcio}

La determinación del Calcio se la realizó utilizando un espectrofotómetro, en el cual se tomó $5 \mathrm{ml}$ de suero de leche en un matraz, se adicionó $50 \mathrm{ml}$ de ácido tricloroacético al $24 \%$ y agua desionizada, esta mezcla fue agitada de forma moderada cada 5 minutos por 30 minutos. Luego se filtra la disolución y se desechó la parte sólida. Luego se toma una muestra de $5 \mathrm{ml}$ de la disolución y se le añadió $1 \mathrm{ml}$ de cloruro de lantano. Esta mezcla fue llevada al equipo para su medición por triplicado. 


\section{Lactosa}

La lactosa se midió siguiendo la norma AOAC 984.15, método enzimático mediante la hidrólisis de lactosa.

pH

La determinación del $\mathrm{pH}$ en el suero se lo realizó mediante el uso de un medidor digital previamente calibrado, mismo que es introducido en la muestra directamente.

\section{Densidad}

Se la determinó con el uso de un termo lactodensímetro Quevenne 15-40 que fue introducido en las muestras de suero.

\section{Coliformes}

Estos microrganismos fueron evaluados mediante el método de vertido en placa siguiendo la norma ISO 9308-1:2014, se procuró realizar un correcto homogenizado de las muestras, dejándolas incubar por 48 horas a $35^{\circ} \mathrm{C}$.

Con el propósito de identificar de identificar y evaluar las características fisicoquímicas y microbiológica se realizó un estudio transversal y con los datos obtenidos se aplicó un diseño experimental, por consiguiente, se aplicó un análisis de varianza de un factor, para determinar si existen diferencias significativas en esos variables de las diferentes especies.

\section{Resultados.}

Al analizar la cantidad de contenido de extracto seco del suero nos referimos al residuo expresado en porcentaje de peso obtenido después de la efectuada desecación de la leche, está compuesto principalmente por proteínas, lactosa como sustancias de sustancial valor nutritivo, grasas, vitaminas, cenizas, calcio y lactosa, la figura 1 representa los valores porcentuales encontrados en cada una de las especies.

Figura 1

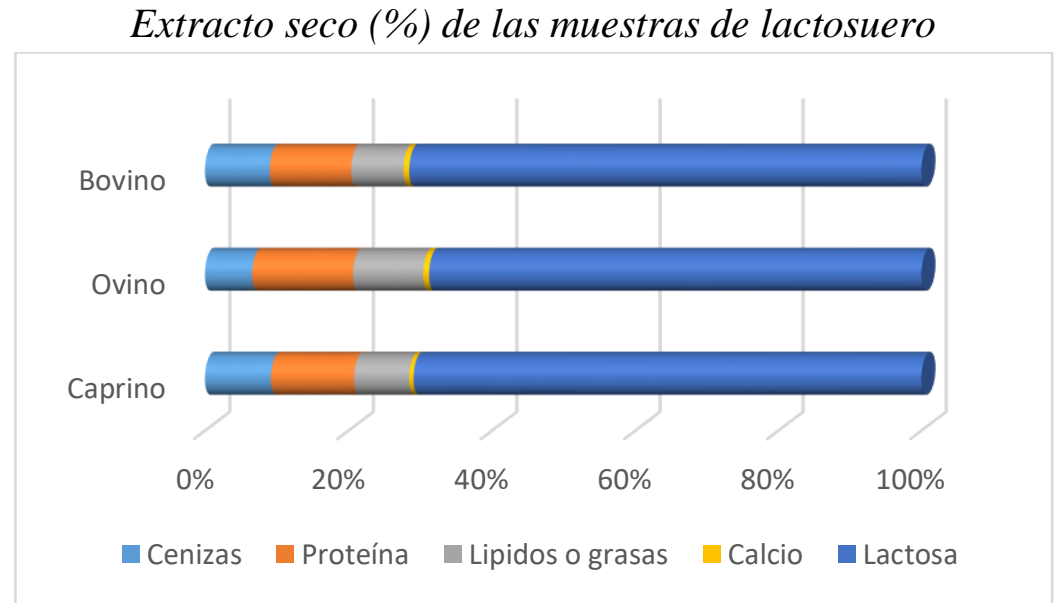

Fuente: Elaboración propia. 
El lactosuero constituye una fuente económica para la industria alimentaria, al contener proteínas que proporcionan diversas propiedades en una extensa gama de alimentos, al referirnos de los derivados del suero (etanol, bebidas no alcohólicas y fermentadas, ácidos orgánicos, aislados, concentrados e hidrolizados de proteína) matizamos la importancia de la lactosa el cual es una propiedad que realza el color y sabor, mejoran la textura y presentan otras propiedades que permiten aumentar la calidad de los alimentos, es por esto la preocupación que genera que se esté utilizando este líquido para alimentar a los cerdos en forma extensiva en los hogares y sectores rurales, además de que una gran parte de este sea desechado en los drenajes y en las quebradas, siendo el suero un alto contaminador del medio ambiente ya que por su $\mathrm{pH}$ acidifica los suelos y provoca inmovilización de metales pesados, en el agua destruye su ecosistema al requerir grandes cantidades de oxígeno para su degradación, además que eutrofiza las aguas con poca corriente.

Tabla 1

Análisis de las propiedades fisicoquímicas y microbiológicas de las muestras de suero

\begin{tabular}{|l|l|l|l|}
\hline \multicolumn{1}{|c|}{ Propiedad } & \multicolumn{1}{c|}{ Caprino } & \multicolumn{1}{c|}{ Ovino } & \multicolumn{1}{c|}{ Bovino } \\
\hline Extracto seco (\%) & $6.64^{\mathrm{b}}$ & $7.48^{\mathrm{a}}$ & $6.34^{\mathrm{b}}$ \\
\hline Cenizas (\%) & $0.61^{\mathrm{a}}$ & $0.498^{\mathrm{c}}$ & $0.58^{\mathrm{b}}$ \\
\hline Proteína (\%) & $0.77^{\mathrm{b}}$ & $1.05^{\mathrm{a}}$ & $0.73^{\mathrm{b}}$ \\
\hline Lípidos o grasas (\%) & $0.51^{\mathrm{b}}$ & $0.73^{\mathrm{a}}$ & $0.47^{\mathrm{b}}$ \\
\hline Calcio (\%) & $0.04^{\mathrm{a}}$ & $0.06^{\mathrm{a}}$ & $0.05^{\mathrm{a}}$ \\
\hline Lactosa (\%) & $4.71^{\mathrm{a}}$ & $5.13^{\mathrm{a}}$ & $4.61^{\mathrm{a}}$ \\
\hline $\mathrm{pH}$ & $6.29^{\mathrm{a}}$ & $6.31^{\mathrm{a}}$ & $6.22^{\mathrm{a}}$ \\
\hline Densidad (g/ml) & $1.003^{\mathrm{a}}$ & $1.058^{\mathrm{a}}$ & $1.115^{\mathrm{a}}$ \\
\hline Coliformes (\%) & $1.89^{\mathrm{a}}$ & $1.31^{\mathrm{b}}$ \\
\hline $\begin{array}{l}\text { Los resultados en la misma fila con diferentes letras en superíndice difieren significativamente } \\
\text { (p } \leq 0.05 \text { ) según la prueba de Tukey. }\end{array}$ \\
\hline
\end{tabular}

Fuente: Elaboración Propia.

La tabla 1 presenta un cuadro de resumen de los resultados obtenidos en cada una de las variables estudiadas en cada especie (caprino, ovino y bovino), en lo que se refiere a la cantidad de contenido en extracto seco del lactosuero de oveja es más alto que el de la vaca y cabra (altamente significativa con un $\mathrm{p}<0.01$ ), por el contrario, este parámetro no presentó diferencias significativas entre la cantidad de extracto seco en las especies caprina y bovina.

El contenido de ceniza en el lactosuero de la oveja es menor que el contenido obtenido de la cabra y la vaca, los valores reportados con anterioridad evidencian diferencias altamente significativas $(\mathrm{p}<0.01)$ entre el suero de la especie caprina respecto a la especie ovina y bovina, así mismo la cantidad de contenido de cenizas en el lactosuero de la vaca y oveja $(\mathrm{p}<0.05)$ presentan diferencias significativas. Estos resultados coinciden con los hallazgos encontrados por Keskin et al. (2004) y Bhosale et al. (2009) respecto a la cantidad de contenido de cenizas encontrada en la leche de vaca, de igual manera respecto a la cantidad de contenido de cenizas encontrado en el lactosuero de caprinos coincide con los resultados reportados en una investigación realizada por Sanmartín (2010), agregando a lo anterior los valores obtenidos del contenido de cenizas en el lactosuero de la leche ovina son similares a los resultados de Adewumi y Olorunnisomo (2009). 
Seguidamente no existieron diferencias significativas $(p>0.05)$ entre las proteínas del lactosuero de las especies caprinas y bovinas, por otro lado, la cantidad de contenido de proteína en la leche de oveja fue mayor en comparación al contenido obtenido en las otras 2 especies con un nivel altamente significativo $(\mathrm{p}<0.01)$. Esta reducción del contenido de proteínas depende de la fase de lactancia y el estado de salud de la ubre, además los resultados del contenido de proteínas de cada especie coinciden con la revisión bibliográfica encontrada en Imran et al. (2008), Abd et al. (2009), Aneja et al (2002) y Pavic et al. (2002). Otro aspecto que se puede destacar con relación al suero de leche de oveja es que está representado por un $17-22 \%$ del total de proteínas.

A continuación, se observa que la cantidad de contenido de grasa en el lactosuero de oveja es más alta que los valores resultantes en las especies bovinas y caprinas de acuerdo con la tabla sus diferencias son altamente significativa $(\mathrm{p}<0.01)$, mientras que el lactosuero de la cabra y la vaca no presentan diferencias significativas. La cantidad de grasa encontrada en la lactosa de cabra fue similar a la citada por Bhosale et al (2009), ahora bien, los hallazgos encontrados en la lactosa de vaca coinciden con la investigación realizada por Mahboba y Zuberir (2007) y Samia et al. (2009), del mismo modo al comparar la cantidad de lactosa producida por la especie ovina en esta investigación es similar a la información encontrada en las investigaciones realizadas por Talevski et al., Adewumi y Olorunnisomo durante el año 2009.

Luego al examinar los resultados de los variables calcio y lactosa demuestran que no hay diferencias significativas entre el contenido obtenido en el lactosuero de las especies caprinas, ovinas y bovinas, en contraste con las investigaciones realizadas por Bylund (1995), Lingathurai et al. (2009) y Sawaya et al. (2009) se puede afirmar que los resultados son similares.

Los resultados referentes al $\mathrm{pH}$ indican que los sueros de las tres especies no fueron significativamente diferentes ( $p>0.05$ ) entre sí, de acuerdo a los valores obtenidos se puede clasificarlas como suero dulce. (Kim et al., 1989; Casper et al.,1998; Boumba et al., 2001). Además, el aumento de contenido de proteínas ayuda a reforzar la densidad, por lo tanto, tampoco existen diferencias significativas entre las especies.

Por último, se observa que el lactosuero de la especie caprina presenta mayor cantidad de coliformes que la cantidad obtenida en la especie ovina y bovina, por esta razón las diferencias son altamente significativas, esto puede estar ocasionado por una alimentación inadecuada o por una manipulación incorrecta antes o después del ordeño, de otra forma se podría indicar que la leche fue tratada incorrectamente durante y después del ordeño, incluidos los procesos de refrigeración y transporte (Haenlein, 1987).

\section{Conclusiones.}

- Las técnicas estadísticas realizadas nos permiten identificar al suero de leche ovino como el de mejor calidad ya que posee la mayor cantidad de sólidos totales con un $7.48 \%$, además es el que mayor cantidad de proteínas aporta con un $1.05 \%$ y estas proteínas son las actualmente solicitadas por la industria alimenticia y de 
cosméticos. Estos resultados coinciden con la revisión de la literatura que indica que el contenido de proteína en el lactosuero de oveja es mayor que en la de cabra o de vaca, es evidente ese contenido varía dependiendo de las especies, adicionalmente se puede afirmar esto está influenciado por la raza, la ingesta de alimentos, la paridad, la etapa de lactancia y el estado de salud de la ubre, además se puede destacar que la leche de oveja tiene una muy buena capacidad de coagulación. Además, las estas propiedades mencionadas anteriormente se suman las grasas con un $0.73 \%$, de acuerdo a la revisión bibliográfica los lípidos de la leche son algo similares a los de la cabra, se diferencian por la presencia de ácidos grasos en mayor proporción en la leche de cabra que en la de oveja.

- Para los niveles de calcio, lactosa, pH y densidad no existen diferencias estadísticas significativas entre los tres tipos de lactosuero. Si lo que se busca es obtener azúcar de la leche se podría utilizar cualquiera de estos sueros con rendimientos similares.

- Microbiológicamente el lactosuero caprino es el que mayor cantidad de coliformes posee $1.89 \mathrm{Log} 10 \mathrm{UFC} \cdot \mathrm{ml}-1$, posee diferencias con los otros sueros, aunque no superan los niveles establecidos por la Norma INEN 719.

- La alta producción de lactosuero a nivel mundial y nacional abre un gran abanico de posibilidades de industrialización de este producto que retiene gran parte de los nutrientes de la leche y se encuentra subutilizado o contaminando el medio ambiente.

\section{Referencias bibliográficas.}

Abd Elrahman, S. M., Ahmad, A. M., El Owni, A. O., \& Ahmed, M. K. (2009). Microbiological and physicochemical properties of raw milk used for processing pasteurized milk in blue nile dairy company (Sudan). Australian Journal of Basic and Applied Sciences, 3(4), 3433-3437.

Adewumi, O. O., \& Olorunnisomo, O. A. (2009). Milk yield and milk composition of West African dwarf, Yankasa and crossbred sheep in southwest of Nigeria. Livestock Research for Rural Development, 21(3), 1-8.

Ahmed, M. I., \& El Zubeir, I. E. (2007). The compositional quality of raw milk produced by some dairy cow's farms in Khartoum State, Sudan. Research Journal of Agriculture and Biological Sciences, 3(6), 902-906.

Aneja, R. P., Mathur, B. N., Chandan, R. C., \& Banerjee, A. K. (2002). Technology of indian milk products: handbook on process technology modernization for professionals, entrepreneurs and scientists. Dairy India Yearbook.

Bhosale, S. S., Kahate, P. A., Kamble, K., Thakare, V. M., \& Gubbawar, S. G. (2009). Effect of lactation on physico-chemical properties of local goat milk. Veterinary world, 2(1), 17. 
Boumba, V.A., Voutsinas, L.P., \& Philippopoulos, C.D. (2001). Composition and nutritional value of commercial dried whey products from feta cheese manufacture. International Journal of Dairy Technology, 54(4), 141-145.

Bylund, G. (1995). Dairy processing handbook. Tetra Pak Processing Systems AB S-221. Sweden. 436.

Casper, J.L., Wendorff, W.L., \& Thomas, D.L. (1998). Seasonal changes in protein composition of whey from commercial manufacture of caprine and ovine specialty cheeses. Journal of Dairy Science, 81(12), 3117-3122.

Estrada Guerrero, R., Lemus Torres, D., Mendoza Anaya, D., \& Rodríguez Lugo, V. (2010). Hidrogeles poliméricos potencialmente aplicables en Agricultura. Revista Iberoamericana de Polímeros, 12(2), 76-87.

Gómez Soto, J. A., \& Sánchez Toro, Ó. J. (2019). Producción de galactooligosacáridos: alternativa para el aprovechamiento del lactosuero. Una revisión. Ingeniería y Desarrollo, 37(1), 129-157.

Haenlein, G. F. W. (1993). Producing quality goat milk. International Journal of Animal Sciences, 8, 85-85.

Huertas, R. A. P. (2009). Lactosuero: importancia en la industria de alimentos. Revista Facultad Nacional de Agronomía-Medellín, 62(1), 4967-4982.

Imran, M., Khan, H., Hassan, S. S., \& Khan, R. (2008). Physicochemical characteristics of various milk samples available in Pakistan. Journal of Zhejiang University Science B, 9(7), 546-551.

Keskin, M., Avşar, Y. K., BiÇER, O., \& Güler, M. B. (2004). A comparative study on the milk yield and milk composition of two different goat genotypes under the climate of the Eastern Mediterranean. Turkish Journal of Veterinary and Animal Sciences, 28(3), 531-536.

Kim, S.H., Morr, C.V., Seo, A., \& Surak, J.G. (1989). Effect of whey pretreatment on composition and functional properties of whey protein concentrate. Journal of Food Science, 54(1), 25-29

Pavic, V., Antunac, N., Mioc, B., Ivankovic, A., \& Havranek, J. L. (2002). Influence of stage of lactation on the chemical composition and physical properties of sheep milk. Czech journal of animal science, 47(2), 80-84.

Primicias. (14 de julio 2021) Prohibición del suero líquido provocaría el desperdicio del $90 \%$ de insumos en la industria lechera. https://www.primicias.ec/noticias/economia/suero-leche-industria-agronomialactosuero/ 
Lin, Y.-S., Huang, K.-S., Yang, C.-H., Wang, C.-Y., Yang, Y.-S., Hsu, H.-C., . . . Tsai, C.-W. (2012). Microfluidic synthesis of microfibers for magnetic-responsive controlled drug release and cell culture. PLoS ONE, 7(3), 1-8.

Lingathurai, S., Vellathurai, P., Vendan, S. E., \& Anand, A. A. P. (2009). A comparative study on the microbiological and chemical composition of cow milk from different locations in Madurai, Tamil Nadu. Indian Journal of Science and Technology, 2(2), 51-54.

Ruiz Estrada, G. (2004). Desarrollo de un Sistema de liberación de fármacos basado en nanopartículas magnéticas recubiertas con Polietilénglicol para el tratamiento de diferentes enfermedades. Madrid: Universidad Autónoma de Madrid. Departamento de Física Aplicada.

Sanmartín Estrada, B. (2010). Aprovechamiento de suero de quesería de origen caprino mediante la obtención de concentrados de proteínas séricas y subproductos de clarificación. Estudio de sus propiedades tecnológicas.

Sawaya, W. N., Safi, W. J., Al-Shalhat, A. F., \& Al-Mohammad, M. M. (1984). Chemical composition and nutritive value of goat milk. Journal of Dairy Science, 67(8), 1655-1659.

Song, J., King, S., Yoon, S., Cho, D., \& Jeong, Y. (2014). Enhanced spinnability of narbon nanotube fibers by surfactant addition. Fiberes and Polymers, 15(4), 762766.

Talevski, G., Čobanova-Vasilevska, R., Srbinovska, S., \& Sireta, Z. (2009). Quality of the sheep milk as a raw material in dairy industry of Macedonia. Biotechnology in animal husbandry, 25(5-6-2), 971-977.

Wulff-Pérez, M., Martín-Rodriguez, A., Gálvez-Ruiz, M., \& de Vicente, J. (2013). The effect of polymer surfactant on the rheological properties of nanoemulsions. Colloid and Polymer Science, 291, 709-716.

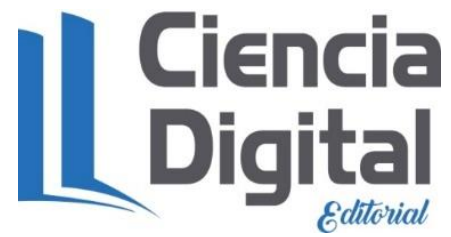




\section{PARA CITAR EL ARTÍCULO INDEXADO.}

Mejía Cabezas, N. T., Campoverde Santos, D. K., \& Velasco Matveev, L. A. (2021). Estudio comparativo de las propiedades fisicoquímicas y microbiológicas de tres tipos de lactosuero (ovino, bovino y caprino) para uso alimentario. ConcienciaDigital, 4(3.1), 301313. https://doi.org/10.33262/concienciadigital.v4i3.1.1831

\section{Liencia}

El artículo que se publica es de exclusiva responsabilidad de los autores y no necesariamente reflejan el pensamiento de la Revista Conciencia Digital.

El artículo queda en propiedad de la revista y, por tanto, su publicación parcial y/o total en otro medio tiene que ser autorizado por el director de la Revista Conciencia Digital.

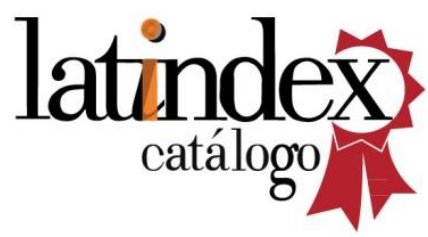

